\title{
Identification of specific modules and significant genes associated with colon cancer by weighted gene co-expression network analysis
}

\author{
YE FENG ${ }^{1}$, YANBO $\mathrm{LI}^{2}, \mathrm{LIN} \mathrm{LI}^{2}$, XUEFENG WANG ${ }^{1}$ and $\mathrm{ZHI} \mathrm{CHEN}{ }^{2}$ \\ ${ }^{1}$ Department of Gastrointestinal Colorectal and Anal Surgery, China-Japan Union Hospital of Jilin University, Changchun, \\ Jilin 130033; ${ }^{2}$ Department of Nephrology, First Hospital of Jilin University, Changchun, Jilin 130021, P.R. China
}

Received January 11, 2018; Accepted March 12, 2019

DOI: $10.3892 / \mathrm{mmr} .2019 .10295$

\begin{abstract}
Colon cancer is one of the most commonly diagnosed malignancies and is a leading cause of cancer-associated mortality. The aim of the present study was to investigate the molecular mechanisms underlying colon cancer and identify potentially significant genes associated with the disease using weighted gene co-expression network analysis (WGCNA). The test datasets used were downloaded from The Cancer Genome Atlas (TCGA) database. WGCNA was applied to analyze microarray data obtained from colon adenocarcinoma samples to identify significant modules and highly associated genes. A gene co-expression network was constructed and different gene modules were selected. Functional and pathway enrichment analyses were performed to investigate the molecular mechanisms of colon cancer. In addition, highly connected hub genes associated with the most significant module were selected for further analysis. Nine specific modules associated with colon cancer were identified, of which the turquoise module was observed to exhibit the greatest association with the disease. Pathway enrichment analysis of the turquoise module suggested that genes in the turquoise module were associated with 'RNA polymerase' and 'purine metabolism'. Furthermore, gene ontology enrichment analysis revealed the top 30 hub genes with a higher degree in the turquoise module, such as $\sigma$-non-opioid intracellular receptor 1 , transmembrane protein 147 TMEM147) and carbamoyl-phosphate synthetase 2, aspartate transcarbamylase, and dihydroorotase, were predominantly enriched in the biological processes 'translation' and 'gene expression'. Experimental verification demonstrated that the expression of TMEM147 in colon cancer was significantly increased compared with the control.
\end{abstract}

Correspondence to: Dr Zhi Chen, Department of Nephrology, First Hospital of Jilin University, 71 Xinmin Street, Changchun, Jilin 130021, P.R. China

E-mail: jichui79xingyou@163.com

Key words: colon cancer, weighted gene co-expression network analysis, significant modules, functional and pathway enrichment analysis, hub genes
Therefore, the results suggested that genes associated with RNA polymerase and the purine metabolic pathways may be substantially involved in the pathogenesis of colon cancer. Furthermore, TMEM147 may represent a biomarker for colon cancer.

\section{Introduction}

Colon cancer is the most common type of gastrointestinal cancer and remains a leading cause of morbidity and mortality worldwide (1). Surgery remains the cornerstone of therapeutic management for patients with early-stage colon cancer (2). At present, there are no effective therapies available for advanced or metastatic colon cancer (3). Recurrence occurs in $10-30 \%$ of patients with colon carcinoma despite the prior use of radical treatment (4,5). A detailed molecular understanding of the pathogenesis of colon cancer is essential for the improvement of clinical outcomes for patients and may reveal additional clinical applications.

Prior studies have aimed to identify genetic alterations involved in the progression of colon cancer in order to provide more effective interventions for the disease (6-8). For example, Wang et al (9) demonstrated that the collagen type XI $\alpha 1$ chain and asporin genes may be involved in the progression of colorectal cancer. Dekervel et al (10) confirmed the involvement of intratumoral hypoxia in the underlying mechanisms of colon cancer and demonstrated the association of hypoxia-driven gene expression with high recurrence rates in advanced colon cancer (10). A recent study indicated that the caudal type homeobox 2 gene may serve as a prognostic biomarker in Stage II and Stage III colon cancer (11). Another recent investigation revealed that overexpression of the Golgi phosphoprotein 3 gene in human colon cancer cells may inhibit cancer cell apoptosis and promote proliferation by activating the Wnt signaling pathway (12). More recently, a study demonstrated that the activity-dependent neuroprotector homeobox gene may represent a pharmacologically inducible repressor of Wnt signaling in colorectal cancer (13). In addition, Fagoonee et al (14) reported that the RNA-binding protein epithelial splicing regulatory protein 1 stimulates the growth of cancer epithelial cells and promotes the progression of human colorectal cancer (14). However, many genes 
associated with the development and progression of colon cancer require further investigation.

Weighted gene co-expression network analysis (WGCNA) groups were applied to functionally categorize genes into modules based on their associations with co-expressed genes (15). Highly interconnected genes (hub genes) in these modules may be involved in the initiation and progression of the associated disease. Liu et al (16) used WGCNA to construct a gene co-expression network and demonstrated that a module associated with the hypertrophic cardiomyopathy pathway was also exhibited in coronary artery disease samples. Liu et al (16) also reported that the glucose-6-phosphate dehydrogenase and S100 calcium binding protein A7 genes may represent potential targets in coronary artery disease (16). In a further study, WGCNA identified seven modules that are notably linked with latent and active tuberculosis (17). Therefore, WGCNA may be applied to analyze microarray data for colon cancer.

In the present study, WGCNA of microarray data obtained from colon cancer samples was performed to further investigate the molecular mechanisms underlying colon carcinoma, and to identify additional potentially significant genes associated with colon cancer. A gene co-expression network was constructed and different gene modules were selected. Functional and pathway enrichment analyses were conducted on genes in the significant modules. Highly connected hub genes in the most significant module were selected, and these genes may be considered to represent candidate biomarkers and therapeutic targets for colon cancer.

\section{Materials and methods}

Microarray data. In the present study, gene expression profile data were downloaded from The Cancer Genome Atlas database (TCGA; https://cancergenome.nih.gov/) (18). The database contained eight colon adenocarcinoma (COAD) $(19,20)$ samples and eight normal samples (https://portal.gdc.cancer. gov), generated in multiple studies.

Data preprocessing. The downloaded sample files were merged into a gene expression matrix. Genes with missing expression values were removed, and the expression values of the remaining genes were $\log _{2}$ transformed. Following preprocessing, the expression matrix with rows and columns contained 14,662 genes and 16 samples.

The downloaded sample data contained data for samples from different batches. The batch differences were removed by batch normalization using the ComBat procedure implemented in the SVA R package (version 1.28.0) (21). Subsequently, the normalize.quantiles.robust function in the preprocess Core package (http://bioconductor. org/packages/release/bioc/html/preprocessCore) was applied to perform unified normalization. Normalized data were used for subsequent analyses, including screening for differences in gene expression and network construction.

Determination of the differential expression levels of genes. Levels of differential gene expression were calculated using the limma R package in Bioconductor (version 3.22.7) (22) via calculation of the $\log _{2}$ fold change (FC) value and the P-value of each gene. Greater $\mid \log _{2} \mathrm{FCl}$ values of genes indicated greater differences in the expression of these genes compared with the normal group and the COAD group. In general, genes with $\log _{2} \mathrm{FC}>1$ and $\mathrm{P}<0.05$ were considered to be upregulated, and genes with $\log _{2} \mathrm{FC}<-1$ and $\mathrm{P}<0.05$ were considered to be downregulated. Furthermore, genes were separated into three categories, according to the $\log _{2} \mathrm{FC}$ value calculated with the limma R package (22). Firstly, genes for which the difference in the expression levels between the disease group and the control group was not significant $\left(-0.5<\log _{2} \mathrm{FC}<0.5\right)$ were excluded from the present study. The remaining genes were separated into two categories for subsequent analysis: i) Genes with $\log _{2} \mathrm{FC} \geq 0.5$; and ii) genes with $\log _{2} \mathrm{FC} \leq-0.5$.

Generation of the WGCNA network, construction of the hierarchical clustering tree and identification of modules. The WGCNA package (version 1.64-1) (http://www.genetics.ucla. edu/labs/horvath/CoexpressionNetwork/Rpackages/WGNA) provides a comprehensive collection of functions for conducting weighted correlation network analysis (23). Instead of describing the correlation structure between thousands of genes and a sample trait, WGCNA analysis focuses on the association between the sample trait and a few, usually $<10$, modules (24). In the WGCNA algorithm, the elements in the co-expression matrix of the genes are no longer the correlation coefficients of the genes, but rather the weighted value of the correlation coefficients. The criteria for the weighted value are such that the connections between the genes contained in each gene network can follow the scale-free law, in which $\mathrm{p}(\mathrm{i})$ is inversely proportional to $\mathrm{i}^{\mathrm{n}}$, where $\mathrm{I}$ is the node degree (connectivity) and $\mathrm{p}(\mathrm{i})$ is the probability that a node has $\mathrm{n}$ links (degree i). In practical applications, the network is made to an approximate scale-free distribution by selecting the weighting coefficients such that $\log (\mathrm{i})$ and $\log [\mathrm{p}(\mathrm{i})]$ are negatively correlated, and the correlation coefficient should be at least 0.8 .

The specific construction process of WGCNA networks includes three steps. In step one, the co-expression matrix of genes is defined. The gene correlation matrix $S=[\mathrm{Smn}]$ is constructed based on the correlation coefficient $\mathrm{Smn}=|\mathrm{cor}(\mathrm{m}, \mathrm{n})|$ between the gene $\mathrm{m}$ and the gene $\mathrm{n}$. In the second step, adjacency functions are defined. In the WGCNA algorithm, for any gene pair, the adjacency coefficient $\mathrm{a}_{\mathrm{m}}$ is used as a measure of inter-gene correlation: $a_{m n}=$ power $\left(S_{m n}, \beta\right)=\left|S_{m n}\right|^{\beta}$. In step three, the parameter $\beta$ of the adjacency function is determined according to the scale-free network principle.

After satisfying the above conditions, the network can be constructed and divided into modules.Linking modules toknown features involves two aspects. The first is the calculation of the characteristic values of the module, followed by the calculation of the correlation coefficient between the feature vector of the module and the feature of interest. The second involves grouped phenotypic data (such as disease status), for which a P-value for each gene for differential expression between each group (e.g. disease and normal groups) is calculated using the t-test, and the $\log _{10}(\mathrm{P}$-value) is defined as gene significance (GS). The module significance (MS) of each module is defined as the mean value of the GS of the genes contained in the module. The MS values are compared. In general, if the MS value of a module is significantly higher than that of other modules, this module may be related to the existence of the disease. 
In the present study, the WGCNA package (24) was used to construct the network and hierarchical clustering tree.

Functional enrichment analysis of significant genes in the identified modules. The Database for Annotation, Visualization and Integrated Discovery (DAVID; http://david. niaid.nih.gov) can help investigators in the functional interpretation of large lists of genes (25). In the present study, gene ontology (GO) and Kyoto Encyclopedia of Genes and Genomes (KEGG) pathway enrichment analyses for important genes in the identified module were performed using the DAVID Bioinformatics Resources (25). P $<0.05$ was set as the criterion for identifying overrepresented GO terms and pathways. In addition, the connection of the genes in the selected module was visualized using Cytoscape software (version 3.1.0) (26).

$R T-q P C R$ validation. Total RNA was extracted from six pairs (female to male ratio, 1:2; mean age, 71; age range, 52-83) of colon cancer tissues and corresponding noncancerous colon tissues, which were collected from the Department of Gastrointestinal Colorectal and Anal Surgery, China-Japan Union Hospital of Jilin University (Changchun, China) using TRIzol ${ }^{\circledR}$ reagent (Invitrogen; Thermo Fisher Scientific, Inc., Waltham, MA, USA), according to the manufacturer's instructions. All participants underwent no other treatment before resection. All tissues were collected between January and April 2018. Harvested tissues were immediately frozen in liquid nitrogen and stored at $-80^{\circ} \mathrm{C}$ prior to RNA extraction. The study was approved by the institutional ethical committee of China-Japan Union Hospital of Jilin University and informed consent was obtained from every patient. The quality and quantity of RNA samples were evaluated using an Infinite M100 PRO microplate reader (Tecan Group, Ltd., Mannedorf, Switzerland). RNA was reverse transcribed to cDNA using a PrimeScript $^{\mathrm{TM}}$ RT Master Mix (Takara Biotechnology Co., Ltd., Dalian, China). All cDNA was amplified using the following primer sets: GAPDH forward, 5'-TGACAACTT TGGTATCGTGGAAGG-3' and reverse, 5'-AGGCAGGGA TGATGTTCTGGAGAG-3'); carbamoyl-phosphate synthetase 2, aspartate transcarbamylase, and dihydroorotase (CAD) forward, 5'-CCATGCACTAGACAGCCAAGA-3' and reverse, 5'-CGGCTCAGTGTGGATACGAC-3'; transmembrane protein 147 (TMEM147) forward, 5'-ACACGCTATGATCTG TACCACA-3' and reverse, 5'-CAGAGGTGGACGAAGGTC TC-3'; and $\sigma$-non-opioid intracellular receptor 1 (SIGMAR1) forward, 5'-CGAAGAGATAGCGCAGTTGG-3' and reverse, 5'-TCCACGATCAGACGAGAGAAG-3'. GAPDH was used as a reference gene for normalization. Power SYBR-Green PCR Master (Thermo Fisher Scientific, Inc.) was used for qPCR, according to the manufacturer's instructions. Each reaction was performed in a final volume of $20 \mu 1$, containing $8 \mu 1$ of cDNA, $1 \mu \mathrm{l}$ of each primer and $10 \mu \mathrm{l} 2 \mathrm{X}$ SYBR Premix EX Taq (Thermo Fisher Scientific, Inc.). RT-qPCR was performed on a Viia7 Real-Time PCR System (Applied Biosystems; Thermo Fisher Scientific, Inc.) using the following thermocycling conditions: Denaturation at $50^{\circ} \mathrm{C}$ for $3 \mathrm{~min}$ and $95^{\circ} \mathrm{C}$ for $3 \mathrm{~min}$; followed by 40 cycles of $95^{\circ} \mathrm{C}$ for $10 \mathrm{sec}$ and $60^{\circ} \mathrm{C}$ for $30 \mathrm{sec}$. Gene expression levels were quantified using the $2^{-\Delta \Delta \mathrm{Cq}}$ method.
Statistical analysis. All data are presented as the mean \pm standard error of the mean and were analyzed using SPSS 22.0 software (SPSS, Inc., Chicago, IL, USA). Differences between colon cancer samples and control samples were determined using the Student's t-test. All experiments were repeated three times. $\mathrm{P}<0.05$ was considered to indicate a statistically significant difference.

\section{Results}

Data preprocessing and differential expression analysis. Following limma analysis, 6,134 genes that were not significantly different between the COAD samples and controls were removed, and 8,528 genes were retained. The 8,528 genes included 4,388 upregulated genes with $\log _{2} \mathrm{FC} \geq 0.5$ in group $\mathrm{A}$ and 4,140 downregulated genes with $\log _{2} \mathrm{FC} \leq-0.5$ in group B. These genes were used for the subsequent analyses.

WGCNA network construction and analysis. The WGCNA package was used to perform gene cluster analysis on the genes $\left(\log _{2} \mathrm{FC} \geq 0.5\right)$ (Fig. 1A). In a cluster dendrogram, height is a measure of dissimilarity according to the topological overlap matrix (23). By selecting different height cutoff values, the gene outliers were screened out and the number of genes was controlled. The genes within the first branch of the hierarchical cluster tree (Fig. 1A) were considered a research target for the follow-up analysis.

WGCNA requires the network to follow the scale-free distribution. As shown in the left panel of Fig. 1B, when $\beta=13$, the network satisfied the scale-free characteristic for the first time, and the vertical axis value exceeded 0.8 (the location of the green line in the figure), which is a prerequisite for building a WGCNA network. The figure on the right of Fig. 1B depicts the average connectivity of the network.

After determining whether the network obeyed the scale-free distribution, the hierarchical clustering tree was constructed and the gene modules were identified. As shown in Fig. 1C, branches of the hierarchical cluster tree defined nine modules with assigned colors. A total of two methods were used to examine the association between each module and colon cancer. The first was the MS value. The gene significance of the genes in each module was calculated. The MS was defined as the mean value of GS. A higher MS value for a module indicated that module had a stronger correlation with the disease. The second method was an MS correlation analysis (27). As shown in Fig. 1D and Table I, the turquoise module displayed the strongest correlation with the disease.

Functional enrichment analysis of genes in the turquoise module. KEGG pathway enrichment analysis was conducted for the genes in the turquoise module (Table II). Genes in this module were mainly related to 'RNA polymerase' and 'purine metabolism'. The top 30 genes with high connectivity were selected from the turquoise module for GO functional annotation. These genes were mainly enriched in the biological processes 'translation' and 'gene expression', in the cellular component 'ribonucleoprotein complex' and in the molecular function 'structural constituent of ribosome' (Table III). The associations of these 30 genes are presented in Fig. 2. The top 30 genes were all upregulated with $\log _{2} \mathrm{FC}>1$, including 
A

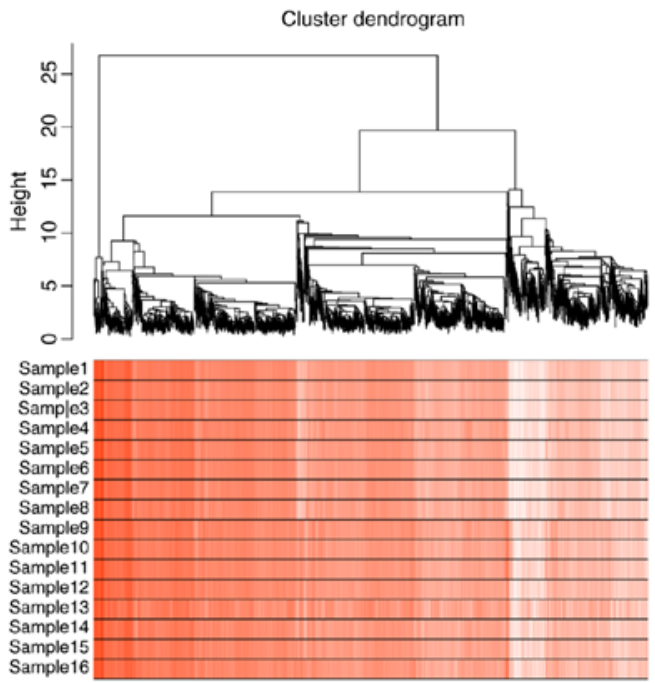

B

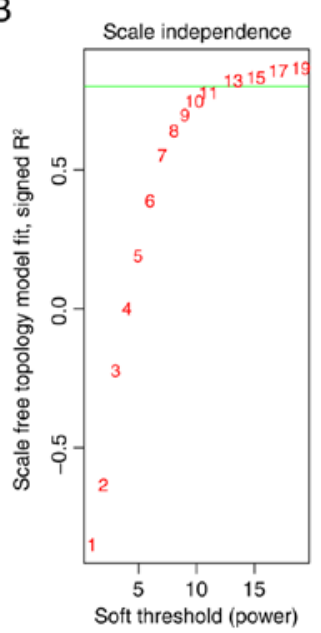

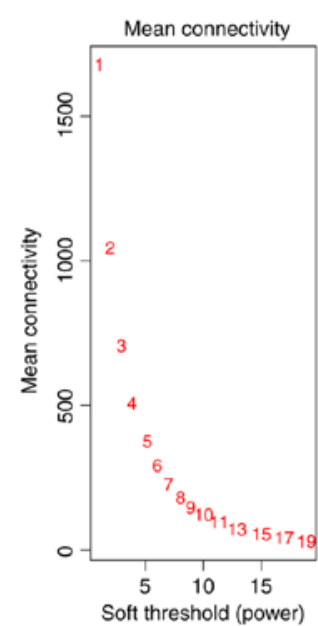

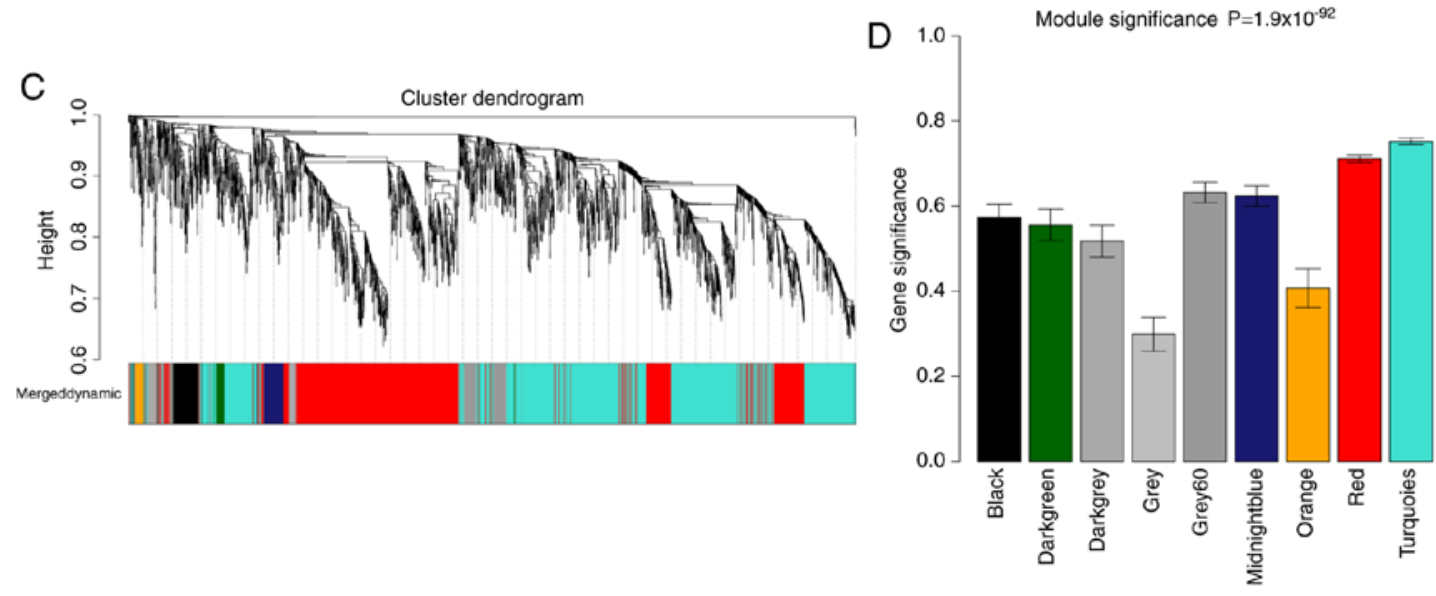

Figure 1. WGCNA. (A) Hierarchical cluster tree generated using the topological overlap measure dissimilarity. (B) Determination of parameter $\beta$ of the adjacency function in the WGCNA algorithm. (C) The hierarchical cluster tree of genes in the colon cancer network. Each color represents a certain gene module. (D) The module significance (average gene significance) of the modules. WGCNA, weighted gene co-expression network analysis.

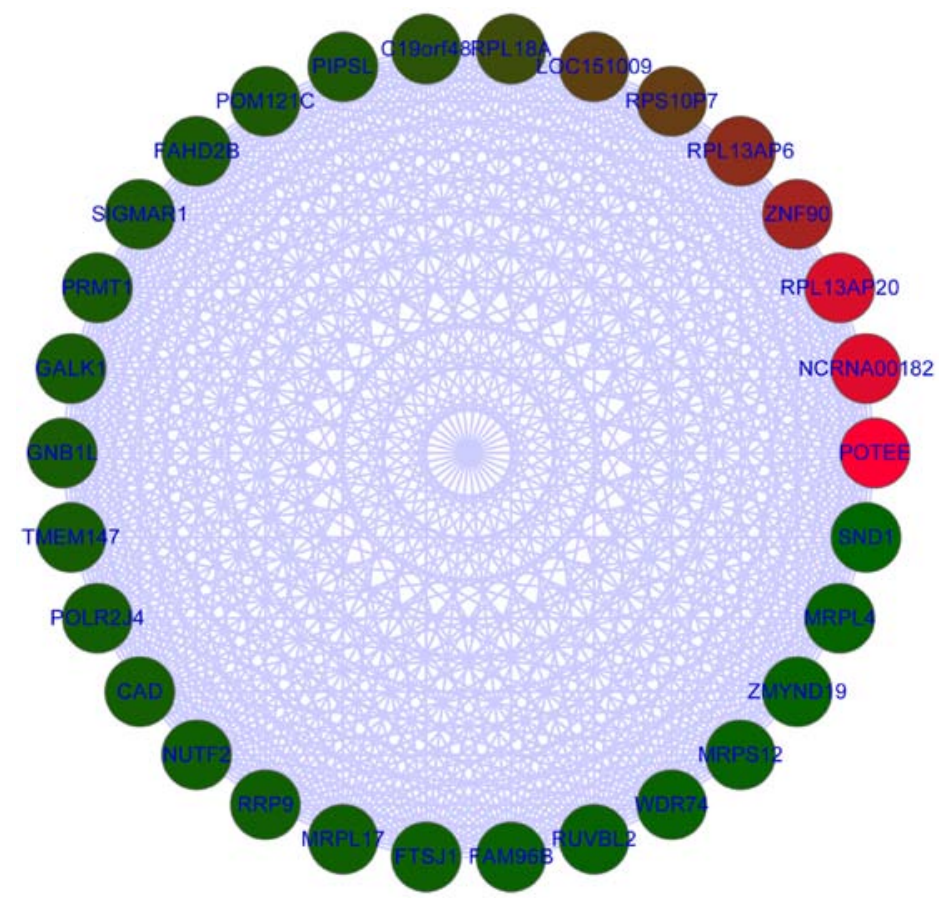

Figure 2. Association of the top 30 genes in the turquoise module, assessed using Cytoscape software. This figure is fully connected and each node is connected with the remaining nodes. Colors from red to green represent the $\log _{2} \mathrm{FC}$ value from large to small. The darker the color, the higher the degree of differential expression. 
POTE ankyrin domain family member E, SIGMAR1, protein arginine methyltransferase 1, galactokinase 1, TMEM147 and CAD.

WGCNA analysis was also performed for genes in group B with $\log _{2} \mathrm{FC} \leq-0.5$. However, the analysis did not meet the condition of constructing a scale-free network and it was not possible to identify significant gene modules for group B.

$R T-q P C R$ validation of identified genes. To validate the gene expression changes identified by the aforementioned bioinformatics analysis, RT-qPCR was used to evaluate a number of potentially critical genes in color cancer, including CAD, TMEM147 and SIGMAR1, which exhibited a high degree in the constructed network. As shown in Fig. 3, the expression of TMEM147 was significantly higher in the colon cancer tissues than in the control tissues. However, no significant differences in the expression of CAD and SIGMAR1 were evident between colon cancer tissues and control tissues, perhaps reflecting the small sample size.

\section{Discussion}

In the present study, nine modules associated with COAD were identified. Of these, the turquoise module exhibited the strongest correlation with the disease. Genes in this module were associated with 'RNA polymerase' and 'purine metabolism'. In addition, the top 30 hub genes with a higher degree in the turquoise module, which included SIGMAR1, TMEM147 and CAD, were predominantly enriched in the biological processes 'translation' and 'gene expression'.

Cancer involves marked changes to the transcription factors associated with RNA polymerases I and III (28). Recently, Bellido et al (29) determined that there is an association between germline mutations in a subunit of the RNA polymerase III transcription complex and a predisposition to colorectal cancer. In addition, purine metabolizing enzymes have been revealed to exhibit increased activities to attenuate accelerated purine metabolism occurring in cancerous tissues (30). Purine antimetabolites are an important class of drugs in the treatment of cancer (31). In the present study, genes in the significant modules were predominantly involved in 'RNA polymerase' and 'purine metabolism', and therefore are important to the pathogenesis of colon cancer.

SIGMAR1 encodes a receptor protein that has been suggested to have an important role in the cellular functions of various tissues associated with the immune, endocrine and nervous systems $(32,33)$. An association between SIGMAR1 and colon cancer has been demonstrated in numerous studies $(34,35)$. SIGMAR1 may induce the invasive abilities of cancer cells by regulating cellular electrical activity in response to extracellular stimulation (36). Recently, Gueguinou et al (34) observed that increased SIGMAR1 expression induces breast and colorectal cancer cell migration by regulating calcium homeostasis. In addition, SIGMAR1 was revealed to be significantly enriched in the turquoise module, which exhibited the highest connection with colon cancer. In conclusion, it was hypothesized that SIGMAR1 may have an important role in the regulation of colon cancer cell migration and invasion. However, further studies using a larger number of samples are required to confirm this finding. 
Table II. KEGG pathway enrichment analysis for genes in the turquoise module.

\begin{tabular}{lllr}
\hline KEGG ID & \multicolumn{1}{c}{ Description } & \multicolumn{1}{c}{ P-value } & \multicolumn{1}{c}{ Count } \\
\hline hsa03020 & RNA polymerase & $4.70 \times 10^{-8}$ & 13 \\
hsa00230 & Purine metabolism & $8.27 \times 10^{-6}$ & 29 \\
hsa00240 & Pyrimidine metabolism & 0.000117076 & 19 \\
hsa03010 & Ribosome & 0.000136386 & 18 \\
hsa03008 & Ribosome biogenesis in eukaryotes & 0.000284171 & 16 \\
hsa00030 & Pentose phosphate pathway & 0.00060088 & 8 \\
hsa01100 & Metabolic pathways & 0.000680717 & 112 \\
hsa03013 & RNA transport & 0.000911011 & 23 \\
hsa00100 & Steroid biosynthesis & 0.002054999 & 6 \\
hsa03040 & Spliceosome & 0.007030765 & 18
\end{tabular}

KEGG, Kyoto Encyclopedia of Genes and Genomes.

Table III. GO analysis of the top 30 genes with higher connectivity.

\begin{tabular}{llrr}
\hline Category & \multicolumn{1}{c}{ Term } & Count & P-value \\
\hline GOTERM_BP_ALL & GO:0006412-translation & 6 & $4.58 \times 10^{-5}$ \\
GOTERM_BP_ALL & GO:0010467-gene expression & 11 & 0.001482883 \\
GOTERM_BP_ALL & GO:0044267-cellular protein metabolic process & 9 & 0.005342118 \\
GOTERM_BP_ALL & GO:0009058-biosynthetic process & 11 & 0.005548833 \\
GOTERM_BP_ALL & GO:0008152-metabolic process & 16 & 0.009967762 \\
GOTERM_CC_FAT & GO:0030529-ribonucleoprotein complex & 8 & $3.58 \times 10^{-6}$ \\
GOTERM_CC_FAT & GO:0005840-ribosome & 6 & $9.21 \times 10^{-6}$ \\
GOTERM_CC_FAT & GO:0033279-ribosomal subunit & 3 & $7.17 \times 10^{-4}$ \\
GOTERM_CC_FAT & GO:0005635-nuclear envelope & 5 & 0.033087058 \\
GOTERM_MF_FAT & GO:0003735-structural constituent of ribosome & 5 & $4.36 \times 10^{-5}$ \\
GOTERM_MF_FAT & GO:0005198-structural molecule activity & 0.006405258
\end{tabular}

GO, gene ontology; BP, biological process; CC, cellular component; MF, molecular function.
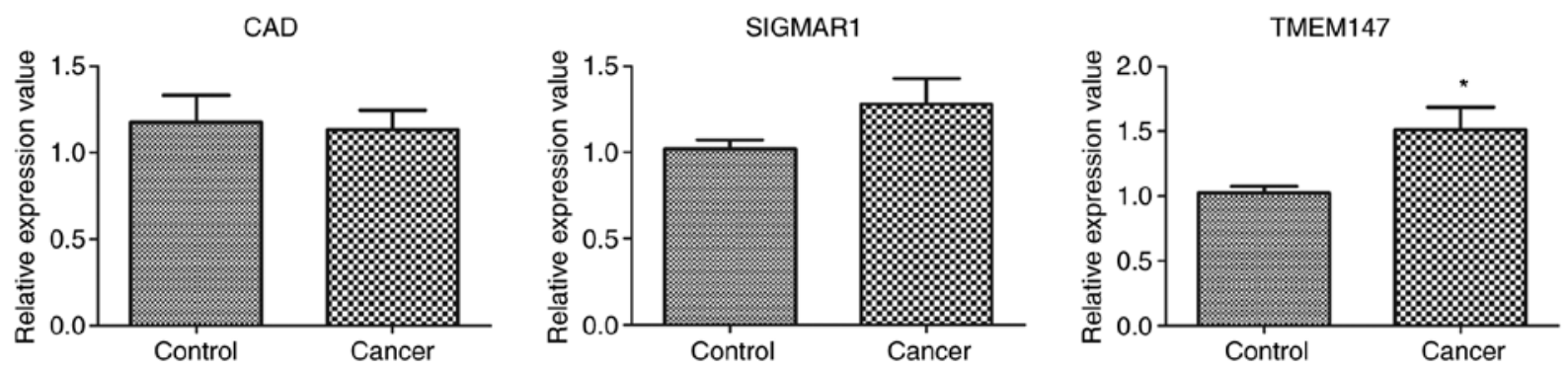

Figure 3. Reverse transcription-quantitative polymerase chain reaction validation of expression changes in three selected differentially expressed genes (CAD, SIGMAR1 and TMEM147) between colon cancer samples and control samples. ${ }^{*} \mathrm{P}<0.05$ vs. respective control (Student's t-test). CAD, carbamoyl-phosphate synthetase 2, aspartate transcarbamylase, and dihydroorotase; SIGMAR1, o-non-opioid intracellular receptor 1; TMEM147, transmembrane protein 147.

TMEM147 is a member of the transmembrane protein family, which has important roles in various physiological processes in disease, including migration, adhesion and signal transduction regulation (37). TMEM147 stimulates cell proliferation via negative modulation of M3 muscarinic receptor expression in H508 human colon cancer cells (38). In the present study, TMEM147 was upregulated and additionally identified as a hub gene in the most significant module associated with colon cancer. Furthermore, the expression level of TMEM147 in colon cancer was investigated using RT-qPCR. Thus, it is conceivable that TMEM147 serves an important role in the development and progression of colon 
cancer. Additional studies are required to assess the role of TMEM147 in colon cancer.

Pathway enrichment analysis of the turquoise module suggested that genes in the turquoise module were related to the purine metabolic pathway. CAD is associated with the enzymatic activities of the first three enzymes in pyrimidine biosynthesis: Carbamoyl phosphate synthetase, aspartate transcarbamoylase, and dihydroorotase (39). The multifunctional CAD protein can control gene expression in both the nucleus and cytoplasm (40). Additionally, regulation of pyrimidine pathways is disrupted in malignancies (41). Purine and pyrimidine antimetabolites remain an important class of drugs for the treatment of cancer (42). In the present study, CAD was a hub gene in the significant module associated with colon cancer. The results support the potentially significant role of $\mathrm{CAD}$ in the pathogenesis of colon cancer. This possibility is currently being assessed.

Nevertheless, the present study had some limitations. Firstly, relatively few samples were included for the bioinformatics analysis, which may have affected the accuracy of the WGCNA analysis. More gene datasets should be used for cross-validation. Secondly, CAD and SIGMAR1 did not exhibit significant differences between the colon cancer tissues and control tissues, which may be affected by the small sample size. However, the roles of CAD and SIGMAR1 merit future experimental verification in studies with more samples. Thirdly, the top 30 hub genes with a high degree in the turquoise module were illustrated in the present study; only three of these were selected as the primary targets, and the other genes also merit further investigation.

In conclusion, nine specific modules were identified in COAD samples, of which the turquoise module displayed the highest correlation with the disease. Genes associated with 'RNA polymerase' and 'purine metabolism' may be significantly involved in the pathogenesis of colon cancer. TMEM147 may play an important role in the development and progression of colon cancer. Experimental verification with a larger number of samples is required to verify the results. Additionally, the roles of CAD and SIGMAR1 warrant future experimental verification in studies with more samples. The increased understanding of the role of genetic alterations will provide novel insights into the mechanisms of colon cancer, and may be critical for the development of a future therapeutic intervention for colon cancer.

\section{Acknowledgements}

Not applicable.

\section{Funding}

The present study was partly supported by the Technology Research and Development Program of Jilin Province (grant nos.2013C014-2 and2014Y083), and theScience and Technology Development Program (grant no. 20140204028XY).

\section{Availability of data and materials}

The datasets used and/or analyzed during the current study are available from the corresponding author on reasonable request.

\section{Authors' contributions}

$\mathrm{YF}$ and $\mathrm{ZC}$ were responsible for the conception and design of the research, and drafting the manuscript. YL performed the data acquisition. LL performed the data analysis and interpretation. XW and YF participated in the design of the study and performed the statistical analysis. All authors have read and approved the manuscript.

\section{Ethics approval and consent to participate}

All procedures were approved by the institutional ethical committee of China-Japan Union Hospital of Jilin University. All patients provided informed consent before the study.

\section{Patient consent for publication}

Informed consent was obtained.

\section{Competing interests}

The authors declare that they have no competing interests.

\section{References}

1. Torre LA, Bray F, Siegel RL, Ferlay J, Lortet-Tieulent J and Jemal A: Global cancer statistics, 2012. CA Cancer J Clin 65: 87-108, 2015

2. Bertelsen CA, Neuenschwander AU, Jansen JE, Wilhelmsen M, Kirkegaard-Klitbo A, Tenma JR, Bols B, Ingeholm P, Rasmussen LA, Jepsen LV, et al: Disease-free survival after complete mesocolic excision compared with conventional colon cancer surgery: A retrospective, population-based study. Lancet Oncol 16: 161-168, 2015.

3. Tauriello DV, Calon A, Lonardo E and Batlle E: Determinants of metastatic competency in colorectal cancer. Mol Oncol 11: 97-119, 2017.

4. Kantara C, O'connell MR, Luthra G, Gajjar A, Sarkar S, Ullrich RL and Singh P: Methods for detecting circulating cancer stem cells (CCSCs) as a novel approach for diagnosis of colon cancer relapse/metastasis. Lab Invest 95: 100-112, 2015.

5. Tie J, Wang Y, Tomasetti C, Li L, Springer S, Kinde I, Silliman N, Tacey M, Wong HL, Christie M, et al: Circulating tumor DNA analysis detects minimal residual disease and predicts recurrence in patients with stage II colon cancer. Sci Transl Med 8: 346ra92, 2016.

6. Gröne J, Weber B, Staub E, Heinze M, Klaman I, Pilarsky C, Hermann K, Castanos-Velez E, Röpcke S, Mann B, et al: Differential expression of genes encoding tight junction proteins in colorectal cancer: Frequent dysregulation of claudin-1, -8 and -12. Int J Colorectal Dis 22: 651-659, 2007.

7. Solé X, Crous-Bou M, Cordero D, Olivares D, Guinó E, Sanz-Pamplona R, Rodriguez-Moranta F, Sanjuan X, de Oca J, Salazar R and Moreno V: Discovery and validation of new potential biomarkers for early detection of colon cancer. PLoS One 9: e106748, 2014.

8. Bhat A, Pope J, Smith J, Ahmad R, Chen X, Washington M, Beauchamp R, Singh A and Dhawan P: Claudin-7 expression induces mesenchymal to epithelial transformation (MET) to inhibit colon tumorigenesis. Oncogene 34: 4570-4580, 2015.

9. Wang J, Yu H, Ye L, Jin L, Yu M and Lv Y: Integrated regulatory mechanisms of miRNAs and targeted genes involved in colorectal cancer. Int J Clin Exp Pathol 8: 517-529, 2015.

10. Dekervel J, Hompes D, van Malenstein H, Popovic D, Sagaert X, De Moor B, Van Cutsem E, D'hoore A, Verslype C and van Pelt J: Hypoxia-driven gene expression is an independent prognostic factor in stage II and III colon cancer patients. Clin Cancer Res 20: 2159-2168, 2014.

11. Dalerba P, Sahoo D, Paik S, Guo X, Yothers G, Song N, Wilcox-Fogel N, Forgó E, Rajendran PS, Miranda SP, et al: CDX2 as a prognostic biomarker in stage II and stage III colon cancer. N Engl J Med 374: 211-222, 2016. 
12. Qiu CZ, Wang MZ, Yu WS, Guo YT, Wang CX and Yang XF: Correlation of GOLPH3 gene with Wnt signaling pathway in human colon cancer cells. J Cancer 7: 928-934, 2016.

13. Blaj C, Bringmann A, Schmidt EM, Urbischek M, Lamprecht $S$, Fröhlich T, Arnold GJ, Krebs S, Blum H, Hermeking H, et al: ADNP is a therapeutically inducible repressor of WNT signaling in colorectal cancer. Clin Cancer Res 23: 2769-2780, 2017.

14. Fagoonee S, Picco G, Orso F, Arrigoni A, Longo DL, Forni M, Scarfò I, Cassenti A, Piva R, Cassoni P, et al: The RNA-binding protein ESRP1 promotes human colorectal cancer progression. Oncotarget 8: 10007-10024, 2017.

15. Gao B, Shao Q, Choudhry H, Marcus V, Dong K, Ragoussis J and Gao ZH: Weighted gene co-expression network analysis of colorectal cancer liver metastasis genome sequencing data and screening of anti-metastasis drugs. Int J Oncol 49: 1108-1118, 2016

16. Liu J, Jing L and Tu X: Weighted gene co-expression network analysis identifies specific modules and hub genes related to coronary artery disease. BMC Cardiovasc Disord 16: 54, 2016.

17. Jha M, Malhotra AG, Singh S and Pandey KM: Gene co-expression network analysis reveals common system-level properties of genes involved in tuberculosis across independent gene expression studies. Netw Model Anal Health Inform Bioinforma 5: 24, 2016

18. Tomczak K, Czerwinska P and Wiznerowicz M: The cancer genome atlas (TCGA): An immeasurable source of knowledge. Contemp Oncol (Pozn) 19: A68-A77, 2015.

19. Sun D, Chen J, Liu L, Zhao G, Dong P, Wu B, Wang J and Dong L: Establishment of a 12-gene expression signature to predict colon cancer prognosis. PeerJ 6: e4942, 2018.

20. Chen XY, Zhang J, Hou LD, Zhang R, Chen W, Fan HN Huang YX, Liu H and Zhu JS: Upregulation of PD-L1 predicts poor prognosis and is associated with miR-191-5p dysregulation in colon adenocarcinoma. Int J Immunopathol Pharmacol 32: 2058738418790318, 2018

21. Johnson WE, Li C and Rabinovic A: Adjusting batch effects in microarray expression data using empirical Bayes methods. Biostatistics 8: 118-127, 2007.

22. Ritchie ME, Phipson B, Wu D, Hu Y, Law CW, Shi W and Smyth GK: limma powers differential expression analyses for RNA-sequencing and microarray studies. Nucleic Acids Res 43: e47, 2015 .

23. Langfelder $P$ and Horvath S: WGCNA: An R package for weighted correlation network analysis. BMC Bioinformatics 9: 559, 2008.

24. Horvath S and Dong J: Geometric interpretation of gene coexpression network analysis. PLoS Comput Biol 4: e1000117, 2008

25. Huang DW, Sherman BT, Tan Q, Kir J, Liu D, Bryant D, Guo Y, Stephens R, Baseler MW, Lane HC and Lempicki RA: DAVID bioinformatics resources: Expanded annotation database and novel algorithms to better extract biology from large gene lists. Nucleic Acids Res 35: W169-W175, 2007.

26. Kohl M, Wiese S and Warscheid B: Cytoscape: Software for visualization and analysis of biological networks. Methods Mol Biol 696: 291-303, 2011.

27. Ma C, Lv Q, Teng S, Yu Y, Niu K and Yi C: Identifying key genes in rheumatoid arthritis by weighted gene co-expression network analysis. Int J Rheum Dis 20: 971-979, 2017.

28. White RJ: RNA polymerase III transcription and cancer. Oncogene 23: 3208-3216, 2004.
29. Bellido F, Sowada N, Mur P, Lázaro C, Pons T, Valdés-Mas R, Pineda M, Aiza G, Iglesias S, Soto JL, et al: Association between germline mutations in BRF1, a subunit of the RNA polymerase III transcription complex, and hereditary colorectal cancer. Gastroenterology 154: 181-194, 2018.

30. Ozturk HS, Karaayvaz M, Kacmaz M, Kavutcu M, Akgül H and Durak I: Activities of the enzymes participating in purine and free-radical metabolism in cancerous human colorectal tissues. Cancer Biochem Biophys 16: 157-168, 1998.

31. Parker WB: Enzymology of purine and pyrimidine antimetabolites used in the treatment of cancer. Chem Rev 109: 2880-2893, 2009.

32. Belzil VV, Daoud H, Camu W, Strong MJ, Dion PA and Rouleau GA: Genetic analysis of SIGMAR1 as a cause of familial ALS with dementia. Eur J Hum Genet 21: 237-239, 2013.

33. Bernard-Marissal N, Médard JJ, Azzedine H and Chrast R Dysfunction in endoplasmic reticulum-mitochondria crosstalk underlies SIGMAR1 loss of function mediated motor neuron degeneration. Brain 138: 875-890, 2015.

34. Gueguinou M, Crottès D, Chantôme A, Rapetti-Mauss R, Potier-Cartereau M, Clarysse L, Girault A, Fourbon Y, Jézéquel P, Guérin-Charbonnel C, et al: The SigmaR1 chaperone drives breast and colorectal cancer cell migration by tuning SK3-dependent $\mathrm{Ca}^{2+}$ homeostasis. Oncogene 36: 3640-3647, 2017.

35. Sun B, Kawahara M, Ehata S and Nagamune T: AAG8 promotes carcinogenesis by activating STAT3. Cell Signal 26: 1863-1869, 2014.

36. Crottès D, Rapetti-Mauss R, Alcaraz-Perez F, Tichet M, Gariano G, Martial S, Guizouarn H, Pellissier B, Loubat A, Popa A, et al: SigmaR1 regulates membrane electrical activity in response to extracellular matrix stimulation to drive cancer cell invasiveness. Cancer Res 76: 607-618, 2016.

37. Yu F, Ng SS, Chow BK, Sze J, Lu G, Poon WS, Kung HF and Lin MC: Knockdown of interferon-induced transmembrane protein 1 (IFITM1) inhibits proliferation, migration, and invasion of glioma cells. J Neurooncol 103: 187-195, 2011.

38. Rosemond E, Rossi M, McMillin SM, Scarselli M, Donaldson JG and Wess J: Regulation of $\mathrm{M}_{3}$ muscarinic receptor expression and function by transmembrane protein 147. Mol Pharmacol 79: 251-261, 2011.

39. Khan S, Abdelrahim M, Samudio I and Safe S: Estrogen receptor/ Spl complexes are required for induction of cad gene expression by 17 beta-estradiol in breast cancer cells. Endocrinology 144: 2325-2335, 2003

40. Wilkinson MF and Shyu AB: Multifunctional regulatory proteins that control gene expression in both the nucleus and the cytoplasm. Bioessays 23: 775-787, 2001.

41. Löffler M, Fairbanks LD, Zameitat E, Marinaki AM and Simmonds HA: Pyrimidine pathways in health and disease. Trends Mol Med 11: 430-437, 2005.

42. Evans ME, Jones DP and Ziegler TR: Glutamine inhibits cytokine-induced apoptosis in human colonic epithelial cells via the pyrimidine pathway. Am J Physiol Gastrointest Liver Physiol 289: G388-G396, 2005. 